\title{
SYSTEMS OF ISLANDS WITH CONTINUOUS HEIGHT FUNCTIONS
}

\author{
ZSOLT LENGVÁRSZKY \\ (Received 13 June 2011; accepted 25 May 2012; first published online 1 May 2013) \\ Communicated by L. Batten
}

\begin{abstract}
We investigate island systems with continuous height functions and strongly laminar systems which are laminar systems containing sets with disjoint boundaries. In the discrete case, we show that for a maximal rectangular system of islands $\mathcal{H}$ on an $m$ by $n$ rectangular grid we have $\lceil\min (m, n) / 4\rceil \leq|\mathcal{H}| \leq\lceil m / 2\rceil\lceil n / 2\rceil$. In the continuous case we show that under some conditions maximal strongly laminar systems $\mathcal{H}$ have cardinality $\boldsymbol{\aleph}_{0}$ or $2^{\aleph_{0}}$ and present examples with $|\mathcal{H}|=\aleph_{0}$.
\end{abstract}

2010 Mathematics subject classification: primary 05A05; secondary 05A16, 26A03, 26 A30.

Keywords and phrases: systems of islands, continuous height functions, strongly laminar systems.

\section{Introduction}

The general notion of systems of islands was recently defined by Pach et al. [13]. Given a set $\Omega \subseteq \mathbb{R}^{n}, \mathcal{S} \subseteq \mathcal{P}(\Omega)$, and a height function $h: \Omega \rightarrow \mathbb{R}$, a set $H \in \mathcal{S}$ is called an $h$-island in $\mathcal{S}$ if there is an open (in the relative topology) set $G \subseteq \Omega$ such that for cl $H$, the closure of $H$, we have $\mathrm{cl} H \subseteq G$ and for every $x \in G \backslash H$ we have $h(x)<\inf _{H} h$. A collection $\mathcal{H}$ of sets in $\mathcal{S}$ is called a system of islands in $\mathcal{S}$ if $\mathcal{H}$ is the set of all $h$-islands in $\mathcal{S}$ for some height function $h$.

The original concept of systems of rectangular islands in a discrete sense was introduced by Czédli [2] with motivations in coding theory (see Földes and Singhi [4]). Czédli's main result is the formula $f(m, n)=\lfloor(m n+m+n-1) / 2\rfloor$ for the maximal size of systems of islands on an $m$ by $n$ rectangular grid. His techniques involved weak independence, a lattice theory concept that first appeared in Czédli et al. [3]. Baráth et al. [1] found two elementary proofs, one using rooted binary trees, and another using simple mathematical induction. Furthermore, they proved results similar to Czédli's on cylindrical (height $m$, circumference $n$ ), as well as on toroidal ( $m$ by $n$ ) grids, and also in the case of $n$-dimensional hypercubes. They showed that the maximum sizes

(c) 2013 Australian Mathematical Publishing Association Inc. 1446-7887/2013 \$16.00 
of rectangular systems of islands in these cases are $c(m, n)=\lfloor(m+1) n / 2\rfloor, t(m, n)=$ $\lfloor m n / 2\rfloor$, and $b(n)=1+2^{n-1}$, respectively. A triangular analogue of rectangular island systems appeared in Horváth et al. [6], and also in [10]; and brick island systems in higher dimension were examined in Pluhár [14]. Both [6, 14] apply techniques similar to those introduced in Czédli [2]. In [6] the maximum size of triangular systems of islands is shown to be $\left(n^{2}+3 n\right) / 5 \leq f(n) \leq\left(3 n^{2}+9 n+2\right) / 14$, and in [14] it is proved that the maximum size of systems of $d$-dimensional bricks on a $d$-dimensional $m_{1} \times m_{2} \times \cdots \times m_{d}$ table satisfies

$$
\begin{aligned}
& \left(m_{1} m_{2} \cdots m_{d}+\sum_{1 \leq j_{1}<j_{2}<\cdots<j_{d-1} \leq d} m_{j_{1}} m_{j_{2}} \cdots m_{j_{d-1}}\right) /\left(2^{d-1}\right)-1 \\
& \quad \leq f\left(m_{1}, m_{2}, \ldots, m_{d}\right) \\
& \quad \leq\left(m_{1}+1\right)\left(m_{2}+1\right) \cdots\left(m_{d}+1\right) /\left(2^{d-1}\right)-1 .
\end{aligned}
$$

Island systems with square islands were investigated in Horváth et al. [5] and in [9].

The general concept of systems of islands by Pach et al. [13] includes all these discrete island systems as well. For example, Czédli's rectangular islands can be obtained by letting $\Omega$ be the $m$ by $n$ rectangle with vertices at $(0,0),(m, 0),(m, n)$, and $(0, n)$ in the plane and by letting $\mathcal{S}$ be the set of rectangles with sides parallel to the coordinate axes and vertices of integer coordinates. The connection to real islands in a geographical sense can be made in an obvious manner, by letting $\mathcal{S}$ be the set of closed and connected subsets of an open connected set $\Omega$ in the plane.

Pach et al. [13] consider laminar systems as well. A collection $\mathcal{H}$ of sets is called laminar if for every pair of sets $H_{1}, H_{2} \in \mathcal{H}$, either $H_{1} \subseteq H_{2}$ or $H_{2} \subseteq H_{1}$ or $H_{1} \cap H_{2}=\emptyset$. A system of islands that are connected sets is always laminar (see [13]). For discrete islands, being a system of islands is in essence equivalent to laminarity. In general, this is not true as shown by Pach et al. [13]. They show, however, that for bounded connected sets in $\mathbb{R}^{n}$, a countable maximal laminar system $\mathcal{H}$ is also a (maximal) system of islands if and only if the distance of any two disjoint sets in $\mathcal{H}$ is positive. Pach et al. [13] also give a general condition under which the size of a maximal laminar system is either countable or continuum, and exhibit an example consisting of countably many circles in the plane that form a maximal system of islands, answering a question in [11].

Additional recent investigations of island systems include [7] by Horváth, Šešelja, and Tepavčević, where a link between islands and fuzzy relations is explored; and [12] by Máder and Makay, who examine island systems with height functions that may assume only a fixed set of finitely many values.

Continuity for a system of islands or laminar systems is relevant in two different ways. First, one may consider the continuity of $\mathcal{S}$, that is, when the sets in $\mathcal{S}$ are allowed to change in some continuous manner. This was the focus of [11] and the paper by Pach et al. [13]. Secondly, we may consider the continuity of the height function, and this is the focus of the present paper. When the height function is continuous, then it follows, in addition to laminarity, that the boundaries of the islands are disjoint. 
In this paper we investigate systems of islands with continuous height functions and laminar systems with disjoint boundaries when the system $\mathcal{S}$ is discrete and when it is continuous. Our main concern is the size of maximal systems, and we prove results analogous to those given in $[2,8,11,13]$.

Standard notations will be used throughout the paper: for a set $S$, int $S$ denotes the interior, $\partial S$ denotes the boundary, and $\operatorname{cl} S$ denotes the closure of $S$.

\section{Systems of islands and laminar systems}

The following Lemma shows that systems of islands satisfy a property stronger than laminarity (see Pach et al. [13, Proposition 5]).

Lemma 2.1. Let $\Omega$ be a set in $\mathbb{R}^{n}, \mathcal{S} \subseteq \mathcal{P}(\Omega)$ be a collection of subsets of $\Omega$, and $h: \Omega \rightarrow \mathbb{R}$ be a height function. If $H_{1}$ and $H_{2}$ are h-islands in $\mathcal{S}$ such that $H_{1} \cap H_{2}=\emptyset$ then we have $\mathrm{cl} H_{1} \cap \mathrm{cl} H_{2}=\emptyset$.

Proof. Let us assume $p \in \operatorname{cl} H_{1} \cap \operatorname{cl} H_{2}$ and let $\mathrm{cl} H_{i} \subseteq G_{i} \subseteq \Omega$ be open sets such that for all $x \in G_{i} \backslash H_{i}$ we have $h(x)<\inf _{H_{i}} h(i=1,2)$. It follows that $p$ belongs to both $G_{1}$ and $G_{2}$, and that there exist points $p_{1} \in H_{1} \cap G_{2}$ and $p_{2} \in H_{2} \cap G_{1}$. Since $H_{1}$ and $H_{2}$ are disjoint, we obtain $p_{1} \in G_{2} \backslash H_{2}$ and $p_{2} \in G_{1} \backslash H_{1}$. Then $h\left(p_{1}\right)<\inf _{H_{2}} h \leq h\left(p_{2}\right)$ and $h\left(p_{2}\right)<\inf _{H_{1}} h \leq h\left(p_{1}\right)$, which is a contradiction.

Next we show that if the height function $h$ is continuous, the boundaries of $h$-islands are pairwise disjoint.

Proposition 2.2. Let $\Omega$ be a set in $\mathbb{R}^{n}$, and $\mathcal{S}$ be a collection of connected sets in $\Omega$. If $h: \Omega \rightarrow \mathbb{R}$ is a continuous height function, then every two distinct h-islands in $\mathcal{S}$ have disjoint boundaries.

Proof. Let $H_{1}$ and $H_{2}$ be two distinct $h$-islands in $\mathcal{S}$. The boundaries satisfy $\partial H_{i} \subseteq$ cl $H_{i}(i=1,2)$. Therefore, the statement follows from Lemma 2.1 when $H_{1}$ and $H_{2}$ are disjoint. Let $H_{1} \subset H_{2}, q \in H_{2} \backslash H_{1}$, and assume $p \in \partial H_{1} \cap \partial H_{2}$. Let $\mathrm{cl} H_{i} \subseteq G_{i} \subseteq \Omega$ be open sets such that for all $x \in G_{i} \backslash H_{i}$ we have $h(x)<\inf _{H_{i}} h(i=1,2)$. We can write $H_{2}=\left(H_{2} \backslash \mathrm{cl} H_{1}\right) \cup\left(H_{2} \cap G_{1}\right)$, and since $H_{2}$ is connected, we have $H_{2} \backslash \mathrm{cl} H_{1}=\emptyset$ or $H_{2} \cap G_{1}=\emptyset$ or $\left(H_{2} \backslash \mathrm{cl} H_{1}\right) \cap\left(H_{2} \cap G_{1}\right) \neq \emptyset$.

Assume $H_{2} \backslash \mathrm{cl} H_{1}=\emptyset$. We have $q \in \operatorname{cl} H_{1}$ implying $q \in G_{1}$. Thus $\inf _{H_{2}} h \leq h(q)<$ $\inf _{H_{1}} h$. Let $G$ be any open set containing $p$. Then there exist points $u \in H_{1} \cap G$ and $v \in\left(\Omega \backslash H_{2}\right) \cap\left(G \cap G_{2}\right)$ since $p$ lies in the boundary of both $H_{1}$ and $H_{2}$. It follows that $h(u) \geq \inf _{H_{1}} h>\inf _{H_{2}} h>h(v)$. Therefore, $h$ is not continuous at $p$, which is a contradiction.

The case $H_{2} \cap G_{1}=\emptyset$ is impossible since $p$ lies in the boundary of $H_{2}$ and $G_{1}$ is an open set containing $p$.

Assume there exists a point $r$ in $\left(H_{2} \backslash \mathrm{cl} H_{1}\right) \cap\left(H_{2} \cap G_{1}\right)=\left(H_{2} \backslash \mathrm{cl} H_{1}\right) \cap G_{1}$. We have $\inf _{H_{2}} h \leq h(r)<\inf _{H_{1}} h$ and the same argument as in the first case shows that $h$ would not be continuous at $p$, which again is a contradiction. 
Laminar systems $\mathcal{H}$ with the property that every two distinct sets in $\mathcal{H}$ have disjoint boundaries will be called strongly laminar. Thus, a continuous height function $h$ implies that the system of $h$-islands is strongly laminar. The reverse however is not true.

Example 2.3. If $\Omega=[0,1], \mathcal{S}$ is the set of closed intervals in $[0,1]$, and $\mathcal{H}$ is any strongly laminar system that contains the intervals $H_{0}=\left[\frac{1}{2}, 1\right]$, and $H_{n}=[0, n /(2 n+$ 1)] $(n=1,2, \ldots)$ then $\mathcal{H}$ is not a set of $h$-islands in $\mathcal{S}$ for any continuous height function $h$ on $\Omega$.

Note that 0 is not a boundary point of any of the $H_{n}$, and thus, $\left\{H_{n} \mid n=0,1,2, \ldots\right\}$ is indeed strongly laminar. If $h$ is a height function so that all $H_{n}$ are $h$-islands, then there are open sets $G_{n}$ such that $h(x)<\inf _{H_{n}} h$ for any $x \in G_{n} \backslash H_{n}$. Let $x_{0} \in G_{0} \backslash H_{0}$, and pick $y_{n} \in\left(G_{n} \backslash H_{n}\right) \cap\left[0, \frac{1}{2}\right)$ for $n=1,2, \ldots$ Then $y_{n} \rightarrow \frac{1}{2}$, and for sufficiently large $n$, we have $x_{0} \in H_{n}$. It follows that $h\left(y_{n}\right)<h\left(x_{0}\right)<h\left(\frac{1}{2}\right)$ for $n>n_{0}$, that is, $h$ is not continuous at $\frac{1}{2}$.

The example for a collection of sets which is laminar and not an island system given in Pach et al. [13] is in fact strongly laminar. Thus, strongly laminar systems may fail to be systems of islands for any height functions, not just continuous ones.

On the other hand, for sets on a grid, island systems with continuous height functions and strongly laminar systems are the same. If $\mathcal{H}$ is a strongly laminar collection of rectangles on a grid, we could define $h$ by first considering $h_{0}(x)=\mid\{H \in$ $\mathcal{H} \mid x \in H\} \mid$ and then setting $h(x)=h_{0}(x)$ for all $x$ except when $x$ is a point with distance $0<d(x, H)<\frac{1}{2}$ for an island $H$. For these points we set $h(x)=h_{0}(x)+1-2 d(x, H)$. Then $h$ is continuous and the collection of rectangular $h$-islands on the grid is $\mathcal{H}$.

The following is another basic property of islands with a continuous height function.

Proposition 2.4. Let $\Omega$ be a set in $\mathbb{R}^{n}$, and $\mathcal{S}$ be a collection of sets in $\Omega$. Assume $h: \Omega \rightarrow \mathbb{R}$ is a continuous height function and $H$ is an h-island in $\mathcal{S}$. If $p \in \partial H$ then $h(p)=\inf _{H} h$, and thus, $h$ is constant on $\partial H$.

Proof. Let $H \in \mathcal{S}$ be an $h$-island and $p \in \partial H$. There are sequences $\left(q_{n}\right)$ and $\left(r_{n}\right)$ in $H$ and $\Omega \backslash H$, respectively, so that $\lim _{n \rightarrow \infty} q_{n}=\lim _{n \rightarrow \infty} r_{n}=p$. By the continuity of $h$ we have $\lim _{n \rightarrow \infty} h\left(q_{n}\right)=\lim _{n \rightarrow \infty} h\left(r_{n}\right)=h(p)$. Since $q_{n} \in H$ for all $n$, we have $h\left(q_{n}\right) \geq \inf _{H} h$ for all $n$, implying $h(p)=\lim _{n \rightarrow \infty} h\left(q_{n}\right) \geq \inf _{H} h$. Let $H \subseteq G \subseteq \Omega$ be an open set with the property that $h(x)<\inf _{H} h$ for all $x \in G \backslash H$. For sufficiently large $n$, the points $r_{n}$ lie in $G \backslash H$, and hence, $h\left(r_{n}\right)<\inf _{H} h$ for these $n$, implying $h(p)=\lim _{n \rightarrow \infty} h\left(r_{n}\right) \leq \inf _{H} h$.

\section{Maximal rectangular systems on a rectangular grid}

In this section we examine the size of maximal rectangular island systems with continuous height functions, or equivalently, strongly laminar rectangular systems, on a rectangular grid. These results can be viewed as analogous to those given in Czédli [2] and in [8]. For simplicity, we use an open rectangle for the sea while the islands will be closed rectangles. 
Theorem 3.1. Let $\Omega \subseteq \mathbb{R}^{2}$ be the open rectangle $(-1, m+1) \times(-1, n+1)$, where $m$ and $n$ are positive integers, and let $\mathcal{S}$ be the set of closed rectangles in $\Omega$ having sides parallel to the coordinate axes and vertices with integer coordinates. Assume $\mathcal{H}$ is a maximal rectangular system of islands in $\mathcal{S}$ with a continuous height function (or equivalently, a maximal strongly laminar system in $\mathcal{S}$ ). Then

$$
\left\lceil\frac{\min (m, n)}{4}\right\rceil \leq|\mathcal{H}| \leq\left\lceil\frac{m}{2}\right\rceil\left\lceil\frac{n}{2}\right\rceil,
$$

and both the lower and upper bounds are sharp.

Proof. Let $\mathcal{H}$ be a maximal strongly laminar system in $\mathcal{S}$. We establish the lower bound first, proceeding by induction on $m n$.

The cases when either $m \leq 4$ or $n \leq 4$ are trivial: if, for example, $m \leq 4$, let $H=[0, m] \times[0, n]$ for $m=1,2$, and $H=[1, m-1] \times[0, n]$ for $m=3,4$, and observe that $\mathcal{H}_{0}=\{H\}$ is a maximal strongly laminar system of rectangles in $\mathcal{S}$.

Assume $m \geq 5$ and $n \geq 5$. Suppose the rectangle $H_{0}=[0, m] \times[0, n]$ belongs to $\mathcal{H}$. Then $\mathcal{H}_{0}=\mathcal{H} \backslash\left\{H_{0}\right\}$ is a maximal strongly laminar system in $\mathcal{S}_{0}=\left\{H \in \mathcal{H} \mid S \subseteq \Omega_{0}\right\}$, where $\Omega_{0}=(0, m) \times(0, n)$. Using the inductive hypothesis, we can write

$$
\begin{aligned}
|\mathcal{H}| & =\left|\mathcal{H}_{0}\right|+1 \geq\left\lceil\frac{\min (m-2, n-2)}{4}\right\rceil+1 \\
& =\left\lceil\frac{\min (m, n)}{4}-\frac{1}{2}\right\rceil+1 \geq\left\lceil\frac{\min (m, n)}{4}\right\rceil .
\end{aligned}
$$

Now suppose $H_{0} \notin \mathcal{H}$, and let $H_{1}, \ldots, H_{k}$ be the set of maximal rectangles in $\mathcal{H}$. For each $1 \leq i \leq k$ we let $\mathcal{H}_{i}=\left\{H \in \mathcal{H} \mid H \subseteq H_{i}\right\}$. For each $i$, we replace $\mathcal{H}_{i}$ by $\mathcal{H}_{i}^{\prime}$ as follows. Let $H_{i}$ be $p \times q$. If $\min (p, q) \leq 2$ then we let $\mathcal{H}_{i}^{\prime}=\mathcal{H}_{i}$. If $\min (p, q)>2$, and say $\min (p, q)=p$, we define $\mathcal{H}_{i}^{\prime}$ based on $p \bmod 4$ : let $H_{i}\left(j_{1}, j_{2}\right)$ denote the rectangle that consists of the columns of $H_{i}$ with indices $j_{1}$ through $j_{2}$. If $p \equiv 0 \bmod 4$, then

$$
\mathcal{H}_{i}^{\prime}=\left\{H_{i}(1,1), H_{i}(4,5), H_{i}(8,9), \ldots, H_{i}(p-4, p-3), H_{i}(p, p)\right\}
$$

if $p \equiv 1 \bmod 4$, then

$$
\mathcal{H}_{i}^{\prime}=\left\{H_{i}(1,1), H_{i}(4,5), H_{i}(8,9), \ldots, H_{i}(p-5, p-4), H_{i}(p-1, p)\right\} ;
$$

if $p \equiv 2 \bmod 4$, then

$$
\mathcal{H}_{i}^{\prime}=\left\{H_{i}(1,2), H_{i}(5,6), \ldots, H_{i}(p-1, p)\right\} ;
$$

and if $p \equiv 3 \bmod 4$, then

$$
\mathcal{H}_{i}^{\prime}=\left\{H_{i}(1,1), H_{i}(4,5), H_{i}(8,9), \ldots, H_{i}(p-3, p-2), H_{i}(p, p)\right\} .
$$

Then, using the inductive hypothesis, $\left|\mathcal{H}_{i}\right| \geq 1+\lceil(p-2) / 4\rceil=\lceil p / 4\rceil=\left|\mathcal{H}_{i}^{\prime}\right|$ when $p \equiv 1$ or $2 \bmod 4$, and $\left|\mathcal{H}_{i}\right| \geq 1+\lceil(p-2) / 4\rceil=\lceil p / 4\rceil+1=\left|\mathcal{H}_{i}^{\prime}\right|$ when $p \equiv 0$ or 3 
$\bmod$ 4. With $\mathcal{H}^{\prime}=\bigcup_{i=1}^{k} \mathcal{H}_{i}^{\prime}$ it follows that $\left|\mathcal{H}^{\prime}\right|=\left|\bigcup_{i=1}^{k} \mathcal{H}_{i}^{\prime}\right| \leq|\mathcal{H}|$. Note that the collection of cells $(1 \times 1$ rectangles in $\mathcal{S})$ that have a nonempty intersection with at least one island is the same for $\mathcal{H}_{i}$ and $\mathcal{H}_{i}^{\prime}$. Therefore, $\mathcal{H}^{\prime}$ is a maximal strongly laminar system in $\mathcal{S}$ consisting of rectangles with at least one side having length at most 2. It is now sufficient to prove $\left|\mathcal{H}^{\prime}\right| \geq\lceil\min (m, n) / 4\rceil$.

Let us assume, without loss of generality, that $\min (m, n)=m$. For $K \in \mathcal{H}^{\prime}$, let $K^{+}$be the union of cells in $S$ that have nonempty intersection with $K$. Then, by maximality of $\mathcal{H}^{\prime}$, we obtain $\cup \mathcal{H}^{\prime}=[0, m] \times[0, n]$. Hence, we can write

$$
m n=|[0, m] \times[0, n]| \leq \sum_{K \in \mathcal{H}^{\prime}}\left|K^{+}\right| \leq \sum_{K \in \mathcal{H}^{\prime}} 4 n=4 n\left|\mathcal{H}^{\prime}\right|,
$$

where $|H|$, for a rectangle $H$, denotes the area of $H$. It then follows that $\min (m, n) / 4 \leq$ $\left|\mathcal{H}^{\prime}\right|$, which is equivalent to $\lceil\min (m, n) / 4\rceil \leq\left|\mathcal{H}^{\prime}\right|$.

To see that the lower bound is sharp we define a maximal strongly laminar system $\mathcal{H}$ that consists of 'strips'. Again, assume $\min (m, n)=m$, and define $H_{i}=[4(i-$ $1)+1,4(i-1)+3] \times[0, n]$ for $i=1, \ldots,\lfloor(m+1) / 4\rfloor$. If $m \equiv 0$ or $3 \bmod 4$ then $\mathcal{H}=\left\{H_{i} \mid i=1, \ldots,\lfloor(m+1) / 4\rfloor\right\}$ is maximal and $\lfloor(m+1) / 4\rfloor=\lceil m / 4\rceil$. If $m \equiv 1$ or 2 $\bmod 4$ then $\mathcal{H}=\left\{H_{i} \mid i=1, \ldots,\lfloor(m+1) / 4\rfloor\right\} \cup\{[m-1, m] \times[0, n]\}$ is maximal and $\lfloor(m+1) / 4\rfloor+1=\lceil m / 4\rceil$.

Let us now turn to the upper bound. First we show that the upper bound can be achieved using $1 \times 1$ rectangles: define $\mathcal{K}=\{[2 u, 2 u+1] \times[2 v, 2 v+1] \mid u=$ $0, \ldots,\lceil m / 2\rceil-1 ; v=0, \ldots,\lceil n / 2\rceil-1\}$. Then $\mathcal{K}$ is (maximal) strongly laminar in $\mathcal{S}$ and contains $\lceil m / 2\rceil\lceil n / 2\rceil$ rectangles. This will imply that the upper bound is sharp.

We assume now that $\mathcal{H}$ is a strongly laminar system in $\mathcal{S}$ (without assuming maximality). Again, we proceed by induction on $m n$. The case $m n=1$ is trivial, so we let $m n>1$. Assume $H_{0}=[0, m] \times[0, n] \in \mathcal{H}$. If $m \leq 2$ or $n \leq 2$ then $\mathcal{H}=\left\{H_{0}\right\}$ and $|\mathcal{H}|=1 \leq\lceil m / 2\rceil\lceil n / 2\rceil$ is trivial. If $m>2$ and $n>2$, we apply the inductive hypothesis to $\mathcal{H}_{0}=\mathcal{H} \backslash\left\{H_{0}\right\}$ and $\Omega_{0}=(0, m) \times(0, n)$ to obtain

$$
\begin{aligned}
|\mathcal{H}| & =1+\left|\mathcal{H}_{0}\right| \leq 1+\lceil(m-2) / 2\rceil\lceil(n-2) / 2\rceil \\
& =1+(\lceil m / 2\rceil-1)(\lceil n / 2\rceil-1) \\
& =\lceil m / 2\rceil\lceil n / 2\rceil-\lceil m / 2\rceil-\lceil n / 2\rceil+2 \leq\lceil m / 2\rceil\lceil n / 2\rceil .
\end{aligned}
$$

Assume $H_{0} \notin \mathcal{H}$. Let $H_{1}, \ldots, H_{k}$ be the set of maximal rectangles in $\mathcal{H}$. If $1 \leq i \leq k$ and $H_{i}$ is $p \times q$ then we replace $\mathcal{H}_{i}=\left\{H \in \mathcal{H} \mid H \subseteq H_{i}\right\}$ by $\mathcal{H}_{i}^{\prime}$, where $\mathcal{H}_{i}^{\prime}$ consists of $\lceil p / 2\rceil\lceil q / 2\rceil$ one by one rectangles in $H_{i}$ and forms a (strongly) laminar system in $\{H \in$ $\left.\mathcal{S} \mid H \subseteq H_{i}\right\}$. By the inductive hypothesis, we have $\left|\mathcal{H}_{i}\right| \leq\lceil p / 2\rceil\lceil q / 2\rceil=\left|\mathcal{H}_{i}^{\prime}\right|$. Thus, if $\mathcal{H}^{\prime}=\bigcup_{i=1}^{k} \mathcal{H}_{i}^{\prime}$, then $\left|\mathcal{H}^{\prime}\right| \geq|\mathcal{H}|$ and we will be done if we show $\left|\mathcal{H}^{\prime}\right| \leq\lceil m / 2\rceil\lceil n / 2\rceil$.

Without loss of generality, let $m=\max (m, n) \geq 2$. If $m=2$ then $\left|\mathcal{H}^{\prime}\right| \leq 1=$ $\lceil m / 2\rceil\lceil n / 2\rceil$. Assume $m>2$ and let $H \in \mathcal{H}^{\prime}$ be in the next to last column of $H_{0}=[0, m] \times[0, n]$. Note that $H$ can be shifted along its row to the last column without violating laminarity. Hence, we can assume there are no rectangles in column $m-1$. Write $\mathcal{H}=\mathcal{H}^{(1)} \cup \mathcal{H}^{(2)}$, where $\mathcal{H}^{(1)}=\{H \in \mathcal{H} \mid H \subseteq[0, m-2] \times[0, n]\}$ 
and $\mathcal{H}^{(2)}=\{H \in \mathcal{H} \mid H \subseteq[m-1, m] \times[0, n]\}$ and note that $\mathcal{H}^{(1)}$ and $\mathcal{H}^{(2)}$ are rectangular laminar systems on smaller rectangles, so we can apply the inductive hypothesis:

$$
\begin{aligned}
\left|\mathcal{H}^{\prime}\right| & =\left|\mathcal{H}^{(1)}\right|+\left|\mathcal{H}^{(2)}\right| \\
& \leq\lceil(m-2) / 2\rceil\lceil n / 2\rceil+\lceil 1 / 2\rceil\lceil n / 2\rceil \\
& =(\lceil m / 2\rceil-1)\lceil n / 2\rceil+\lceil n / 2\rceil=\lceil m / 2\rceil\lceil n / 2\rceil .
\end{aligned}
$$

This finishes the proof.

It is easy to see that every integer between the lower and upper bounds will occur as the size of a maximal strongly laminar rectangular system. Using the configuration given in the proof for the upper bound as the initial step, we can 'consolidate' the $1 \times 1$ squares into strips, thereby decreasing the size of $\mathcal{H}$ by 1 at a time. Let us think of the base rectangle as having (essentially) $m$ columns and $n$ rows, and assume without loss of generality that $\min (m, n)=m$. Replace the first $k$ squares in column 1 by a $1 \times(2 k-1)$ rectangle $(1 \times 2 k$ when $n=2 k)$, then we repeat this process in columns $3,5, \ldots, m(-1)$. In the second phase the $1 \times n$ rectangles are consolidated into $2 \times n$ rectangles and/or shifted horizontally, again reducing the size of the maximal laminar system by one at a time, until the configuration for the lower bound is reached.

\section{Continuous systems}

In this section we consider continuous collections $\mathcal{S}$, and examine systems of islands in $\mathcal{S}$ with a continuous height function and strongly laminar systems in $\mathcal{S}$. We do not define continuity of $\mathcal{S}$ formally; instead, we will use conditions that are relevant and/or become interesting when the sets in $\mathcal{S}$ are allowed to change in a continuous fashion. Typical examples include $\mathcal{S}$ being all subintervals of a given interval in $\mathbb{R}$ or all rectangles in the plane or all convex sets in $\mathbb{R}^{n}$.

The main concern regarding island systems and laminar systems in previous investigations has been the size of (maximal) systems, and so is the case in this paper as well as in this section. First we present a continuous height function with no islands.

ExAmple 4.1. Let $\Omega=\mathbb{R}$, and let $\mathcal{S}$ be the set of bounded closed intervals in $\mathbb{R}$. Let the height function $h$ be the Weierstrass function, a function that is continuous everywhere and differentiable nowhere, that is

$$
h(x)=W(x)=\sum_{k=0}^{\infty} a^{k} \cos \left(b^{k} \pi x\right),
$$

where $0<a<1, a b>1+3 \pi / 2$, and $b>1$ is an odd integer. Then the set of $h$-islands in $\mathcal{S}$ is empty.

We use the proof of the fact that $W(x)$ is nowhere differentiable given in Thim [15], where for any fixed $x_{0} \in \mathbb{R}$, two sequences $\left(y_{n}\right)$ and $\left(z_{n}\right)$ are produced with the following properties. 
(i) $y_{n}<x_{0}<z_{n}$ for all $n$.

(ii) $\left(W\left(y_{n}\right)-W\left(x_{0}\right)\right) /\left(y_{n}-x_{0}\right)$ and $\left(W\left(z_{n}\right)-W\left(x_{0}\right)\right) /\left(z_{n}-x_{0}\right)$ have different signs for all $n$.

(iii) $\lim _{n \rightarrow \infty}\left|\left(W\left(y_{n}\right)-W\left(x_{0}\right)\right) /\left(y_{n}-x_{0}\right)\right|=\lim _{n \rightarrow \infty}\left|\left(W\left(z_{n}\right)-W\left(x_{0}\right)\right) /\left(z_{n}-x_{0}\right)\right|=\infty$.

It follows that there are infinite subsequences $\left(y_{n_{k}}\right)$ and $\left(z_{n_{k}}\right)$ such that either (1) $W\left(y_{n_{k}}\right)>W\left(x_{0}\right)$ and $W\left(z_{n_{k}}\right)>W\left(x_{0}\right)$ for all $k$; or (2) $W\left(y_{n_{k}}\right)<W\left(x_{0}\right)$ and $W\left(z_{n_{k}}\right)<$ $W\left(x_{0}\right)$ for all $k$. Let $H=\left[x_{0}, x_{1}\right]$ be any closed interval in $\mathcal{S}$ and let $G$ be an arbitrary open set containing $H$. Assume case (1) and let $k$ be such that $y_{n_{k}} \in G$. Then $W\left(y_{n_{k}}\right)>W\left(x_{0}\right) \geq \inf _{H} W$, so $H$ cannot be an $h$-island in $\mathcal{S}$. Assume case (2) and let $k$ be such that $z_{n_{k}} \in H$. Then by the continuity of $W$ there exists an $l$ such that $y_{n_{l}} \in G$ and $W\left(y_{n_{l}}\right)>W\left(z_{n_{k}}\right) \geq \inf _{H} W$, again showing that $H$ is not an $h$-island in $\mathcal{S}$.

A trivial example with no islands is given by letting $h$ be any monotone function; however, the Weierstrass function $W$ is more versatile: it retains the 'no $W$-island' property for collections $\mathcal{S}$ that contain unbounded intervals and also when $W$ is restricted to any interval $\Omega$ on the real line. In addition, $W$ can be used to obtain a height function $h$ for any given $n \in \mathbb{N}$ so that $\mid\{H \mid H$ is an $h$-island in $\mathcal{S}\} \mid=n$.

ExAmple 4.2. Let $\Omega=\mathbb{R}$, and let $\mathcal{S}$ be the set of bounded closed intervals in $\mathbb{R}$. Set $H_{i}=[4 i, 4 i+2]$ for $i=0,1, \ldots$ For a given $n \in \mathbb{N} \cup\{\infty\}$, define the height function $h$ to be $h(x)=W(0)$ when $x \in \bigcup_{i<n} H_{i}$ and $h(x)=W(x)$ otherwise. Then the $h$-islands are precisely the $H_{i}(i<n)$.

This is immediate from the proof of the nondifferentiability of $W(x)$ given in Thim [15], and from the fact that $W(x)$ attains its maximum if and only if $x$ is an even integer.

One of the main results in Pach et al. [13, Theorem 6] states that under some mild assumptions, the cardinality of any maximal laminar system is countable or continuum. The following theorem is a similar result for strong laminarity.

Theorem 4.3. Let $\mathcal{S}$ be a system of subsets in $\mathbb{R}^{n}$ with finite Lebesgue measure satisfying the following conditions:

(1) $\operatorname{int}(A) \neq \emptyset$ for every $A \in \mathcal{S}$;

(2) if $A, B \in \mathcal{S}$ and $A \nsubseteq B$, then $\lambda(A \backslash B)>0$;

(3) if $C \subseteq \mathcal{S}$ is a chain, then $\bigcap \mathcal{C} \in \mathcal{S}$ or $\lambda(\cap \mathcal{C})=0$.

Then the cardinality of any maximal strongly laminar system in $\mathcal{S}$ is countable or continuum.

Proof. Our proof is similar to that of [13, Theorem 6]. Assume $\mathcal{H}$ is a maximal strongly laminar system in $\mathcal{S}$. First we prove that the cardinality of every maximal chain $C \subseteq \mathcal{H}$ is countable or continuum. Define $R=\lambda(C)=\{\lambda(C) \mid C \in C\}$. Item (2) implies that $C$ and $R$ have the same cardinality, so it is enough to show that $R$ is countable or has cardinality continuum.

We prove that if $x$ is both a left and right limit point of $R$, then $x \in R$. 
Assume $x \notin R$. Let $C^{x}=\{C \in C \mid \lambda(C)>x\}$, and define $C_{0}=\bigcap C^{x}$. We show $C_{0} \in \mathcal{H}$ which in fact will imply $x \in R$. Since $C$ only contains sets with positive Lebesgue measure, and $x$ is a left limit point of $R$, we have $\lambda\left(C_{0}\right)>0$; hence, $C_{0} \in \mathcal{S}$ by (3). Since $C \cup\left\{C_{0}\right\}$ is a chain of sets in $\mathcal{S}$, we need to show $\mathcal{H} \cup\left\{C_{0}\right\}$ is strongly laminar. Suppose $H \cap C_{0} \neq \emptyset$ for some $H \in \mathcal{H}$. Then $H \cap C \neq \emptyset$ for every $C \in C^{x}$. If for all such $C$ we have $H \subseteq C$, then it follows that $H \subseteq C_{0}$. If for some $C \in C^{x}$ we have $C \subseteq H$, then we also have $C_{0} \subseteq H$. Therefore, $\mathcal{H} \cup\left\{C_{0}\right\}$ is laminar.

To show strong laminarity of $\mathcal{H} \cup\left\{C_{0}\right\}$, let us assume for a contradiction, that $p \in \partial C_{0} \cap \partial H$ for some $C_{0} \neq H \in \mathcal{H}$. We need to consider three cases. Assume first $H \cap C_{0}=\emptyset$. Then there must exist a $C \in C^{x}$ such that $H \nsubseteq C$. Since $C \subseteq H$ is not possible, we must have $H \cap C=\emptyset$. For any open set $p \in U$ there exist $q \in H \cap U$ and $r \in C_{0} \cap U$. Since $q \notin C$ and $r \in C$ it follows that $p \in \partial C$, contradicting strong laminarity of $\mathcal{H}$. Assume $C_{0} \subseteq H$. We can not have $H \subseteq C$ for all $C \in C^{x}$, so for some $C \in C^{x}$ we have $C_{0} \subseteq C \subseteq H$. Again, let $p \in U$ be any open set. There exist $q, r \in U$ such that $q \in C_{0}$ and $r \notin H$. It follows that $q \in C$ and $r \notin C$, implying $p \in \partial C$, which contradicts strong laminarity of $\mathcal{H}$.

Now we assume $H \subseteq C_{0}$, and define $C_{x}=\{C \in C \mid \lambda C<x\}$. Since $x$ is a left limit point of $R$ and $\lambda(H)>0$, there is a $C \in C_{x}$ such that $\lambda\left(C_{0} \backslash C\right)<\lambda(H)$. It follows that $C \cap H$ is nonempty, implying $C \subseteq H$ or $H \subseteq C$. The latter would imply $H \subseteq C^{\prime}$ for some $C^{\prime} \in C$ and $p \in \partial H \cap \partial C_{0}$ would yield $p \in \partial C^{\prime}$, contradicting strong laminarity of $\mathcal{S}$. Assume $C \subseteq H$. If for any $C^{\prime} \in C_{x}$ we had $H \subseteq C^{\prime}$, then the previous argument could be applied, so we can assume $C^{\prime} \subseteq H$ for all $C^{\prime} \in C_{x}$. It follows that $H \in C$ and $\lambda(H)=x$, contradicting our assumption.

For any $x \in \operatorname{cl} R \backslash R$ we have either (i) $x=0$, or (ii) $x$ is a right but not a left limit point of $R$, or (iii) $x$ is a left but not a right limit point of $R$. Define a mapping $\phi: \operatorname{cl} R \backslash R \rightarrow \mathbb{Q}$ as follows: in case (i) let $\phi(0)=0$, in case (ii) let $\phi(x)=y<x$ so that $(x-3(x-y), x) \cap R=\emptyset$, and in case (iii) let $\phi(x)=y>x$ so that $(x, x+3(y-$ $x)) \cap R=\emptyset$. Then $\phi$ is one-to-one, so $\operatorname{cl} \backslash R$ is countable. Using the well-known fact that any closed set in $\mathbb{R}^{n}$ is countable or has cardinality continuum, we obtain $R=\operatorname{cl} R \backslash(\operatorname{cl} R \backslash R)$ is countable or has cardinality continuum.

For $r \in \mathbb{R}^{n}$ define $C(r)=\{H \in \mathcal{H} \mid r \in H\}$. Laminarity of $\mathcal{H}$ implies that $C(r)$ is a chain. Let $C^{\prime}(r)$ be a maximal chain in $\mathcal{H}$ containing $C(r)$. Then, since by (1), every set in $\mathcal{S}$ contains a point with rational coordinates, we have $\mathcal{H}=\bigcup_{r \in \mathbb{Q}^{n}} C(r)=\bigcup_{r \in \mathbb{Q}^{n}} C^{\prime}(r)$. If each $C^{\prime}(r)$ in the union is countable, then $\mathcal{H}$ is countable; if there is a $C^{\prime}(r)$ in the union with cardinality continuum, then $\mathcal{H}$ has cardinality continuum.

Since conditions (1)-(3) are the same as those in Pach et al. [13, Theorem 6], the examples (Corollary 7, Proposition 8) for laminarity given in [13] can be used for strong laminarity as well. Hence, we have the following corollary.

Corollary 4.4 .

(1) The cardinality of a maximal strongly laminar system of bounded closed convex sets in $\mathbb{R}^{n}$ with nonempty interior is countable or continuum. 
(2) The cardinality of a maximal strongly laminar system of closed disks in $\mathbb{R}^{n}$ is countable or continuum.

For continuous $\mathcal{S}$, one can find examples $\mathcal{H}$ of (strongly) laminar systems with $|\mathcal{H}|=2^{\aleph_{0}}$ easily; however, examples of maximal (strongly) laminar systems $\mathcal{H}$ with $|\mathcal{H}|=\boldsymbol{\aleph}_{0}$ are more elusive. Finding such an example was the primary motivation in [11]. Pach et al. [13] produced a clever example of a countable maximal laminar system of closed disks in $\mathbb{R}^{2}$, which in fact is also a maximal strongly laminar system. Here we present an alternative method that can be applied to disks and other families of sets as well to find countable maximal laminar and strongly laminar systems.

Given a set $S \subseteq \Omega$ and a system of sets $\mathcal{S} \subseteq \mathcal{P}(\Omega)$, we call a sequence $\left(K_{n}\right)_{n=1}^{\infty}$ of sets an $S$-sequence (in $\mathcal{S}$ ), if (i) $K_{n} \in \mathcal{S}$ for all $n \in \mathbb{N}$; (ii) $K_{n} \subseteq S$ for all $n \in \mathbb{N}$; and (iii) cl $K_{n} \subseteq$ int $K_{n+1}$ for all $n \in \mathbb{N}$. Define $\kappa(S)=\sup \left\{\lambda\left(\cup_{n=1}^{\infty} K_{n}\right) \mid\left(K_{n}\right)\right.$ is an $S$-sequence $\}$. If $\mathcal{H}$ is a strongly laminar system in $\mathcal{S}$, we call a sequence $\left(K_{n}\right)_{n=1}^{\infty}$ of sets in $\mathcal{S}$ an $\mathcal{H}$ sequence (in $\mathcal{S}$ ), if for any $H \in \mathcal{H}$ either $\left(K_{n}\right)$ is an $H$-sequence or $\left(K_{n}\right)$ is an $(\Omega \backslash H)$ sequence. Note that if $\left(K_{n}\right)$ is an $\mathcal{H}$-sequence, then $\mathcal{H} \cup\left\{K_{n} \mid n \in \mathbb{N}\right\}$ is a strongly laminar system in $\mathcal{S}$. We also define $\kappa(\mathcal{H})=\sup \left\{\lambda\left(\bigcup_{n=1}^{\infty} K_{n}\right) \mid\left(K_{n}\right)\right.$ is an $\mathcal{H}$-sequence $\}$.

Proposition 4.5. Let $\Omega$ be a subset of $\mathbb{R}^{m}$ and $\mathcal{S} \subseteq \mathcal{P}(\Omega)$ such that:

(1) int $S \neq \emptyset$ for any $S \in \mathcal{S}$; and

(2) for any open set $S \subseteq \Omega$ there exists an $S$-sequence in $\mathcal{S}$.

Let $\left(\mathcal{H}_{n}\right)_{n=0}^{\infty}$ be a sequence of strongly laminar systems in $\mathcal{S}$ that satisfy the following conditions:

(a) $\mathcal{H}_{n+1}=\mathcal{H}_{n} \cup\left\{K_{i}^{(n)} \mid i \in \mathbb{N}\right\}$, where $\left(K_{i}^{(n)}\right)$ is an $\mathcal{H}_{n}$-sequence such that $\lambda\left(\bigcup_{i=0}^{\infty} K_{i}^{(n)}\right)=\kappa\left(\mathcal{H}_{n}\right)$; and

(b) $\lim _{n \rightarrow \infty} \kappa\left(\mathcal{H}_{n}\right)=0$.

Then $\mathcal{H}=\bigcup_{n=0}^{\infty} \mathcal{H}_{n}$ is a strongly laminar system in $\mathcal{S}$, and if for any $S \in \mathcal{S} \backslash \mathcal{H}$ the set $\mathcal{H} \cup\{S\}$ is a strongly laminar system in $\mathcal{S}$, then either $(\alpha)$ there exists an $H \in \mathcal{H}_{0}$ such that $H \subseteq S$; or there is an $n \in \mathbb{N}$ such that either $(\beta) K_{i}^{(n)} \subseteq S \subseteq K_{i+1}^{(n)}$ for some $i \in \mathbb{N}$, or $(\gamma) K_{i}^{(n)} \subseteq S$ for all $i \in \mathbb{N}$.

Proof. Strong laminarity of $\mathcal{H}$ follows from strong laminarity of $\mathcal{H}_{n}(n \in \mathbb{N})$. Let $\mathcal{H} \cup\{S\}$ be strongly laminar, where $S \in \mathcal{S} \backslash \mathcal{H}$, and assume $H \nsubseteq S$ for all $H \in \mathcal{H}_{0}$. Items (1), (2), (a) and (b) imply that there exists an $n \in \mathbb{N}$ such that $H \nsubseteq S$ for all $H \in \mathcal{H}_{n-1}$ and $H \subseteq S$ for some $H \in \mathcal{H}_{n}$. It follows that $K_{1}^{(n)} \subseteq S$ and if there is a largest $i$ with $K_{i}^{(n)} \subseteq S$ then we must have $K_{i}^{(n)} \subseteq S \subseteq K_{i+1}^{(n)}$, otherwise $K_{i}^{(n)} \subseteq S$ for all $i \in \mathbb{N}$.

Let $\mathcal{S}$ be the set of closed disks in $\mathbb{R}^{2}$. Conditions (1) and (2) of Proposition 4.5 are clearly satisfied. We may define $\mathcal{H}_{0}$ to be the set of disks $H_{i}$ with center at the origin and radius $1+1 / 2+\cdots+1 / i$, and the $\mathcal{H}_{n}$ so that item (a) of the proposition is satisfied. To see the latter we prove the following claim.

Claim. Let $S$ be a set in $\mathbb{R}^{2}$ with finite Lebesgue measure. Then there exists an open disk $D \subseteq S$ such that $\lambda(D)=\kappa(S)$. 
We have $\kappa(S) \leq \lambda(S)<\infty$. Hence, for each $n \in \mathbb{N}$ there exists an $S$-sequence $\left(K_{i}^{(n)}\right)_{i=1}^{\infty}$ in $\mathcal{S}$ with $\lambda\left(\bigcup_{i=1}^{\infty} K_{i}^{(n)}\right)>\kappa(S)-1 / n$. Pick $K_{i_{n}}^{(n)}$ so that $\lambda\left(K_{i_{n}}^{(n)}\right)>\kappa(S)-1 / n$. Then, $\lim _{n \rightarrow \infty} \lambda\left(K_{i_{n}}^{(n)}\right)=\kappa(S)$, and if $c_{n}$ denotes the center of $K_{i_{n}}^{(n)}$, then the sequence $\left(c_{n}\right)_{n}$ must be bounded. Let $\left(c_{n_{k}}\right)_{k}$ be a convergent subsequence and let $c=\lim _{k \rightarrow \infty} c_{n_{k}}$. Then it follows that the open disk $D$ with center at $c$ and Lebesgue measure $\kappa(S)$ is contained in $S$. This proves the claim.

The boundaries of the circles in $\mathcal{H}_{n}$ partition $\mathbb{R}^{2}$ into sets such that only finitely many of them have Lebesgue measure greater than or equal to $\epsilon$ for any $\epsilon>0$. Thus, $\left(K_{i}^{(n)}\right)_{i}$ in item (a) of Proposition 4.5 can be chosen by considering the largest open disk $D$ in any of the finitely many parts in $\mathcal{H}_{n}$ with Lebesgue measure greater than or equal to $\kappa\left(\mathcal{H}_{n}\right)$. If $D$ has center $c$ and radius $r=\kappa\left(\mathcal{H}_{n}\right)$ then we define the $K_{i}^{(n)}$ to be the closed disks with centers at $c$ and radii $r_{i}$ that converge to $r$ in a strictly increasing fashion.

If we pick $K_{1}^{(n)}$ so that $\lambda\left(K_{1}^{(n)}\right)=(1 / 2) \kappa\left(\mathcal{H}_{n}\right)$ for every $n \in \mathbb{N}$, then item (b) will be satisfied as well. Indeed, it should be clear that with $a_{n}=\kappa\left(\mathcal{H}_{n}\right)=\lambda\left(\bigcup_{i=1}^{\infty} K_{i}^{(n)}\right)$, the sequence $\left(a_{n}\right)$ is decreasing. Also, since $\mathcal{H}$ is laminar, for $m>n$, either $a_{m} \leq(1 / 2) a_{n}$ or $\left(\bigcup_{i=1}^{\infty} K_{i}^{(m)}\right) \cap\left(\bigcup_{i=1}^{\infty} K_{i}^{(n)}\right)=\emptyset$. Hence, if $a_{n} \rightarrow 0(n \rightarrow \infty)$ is not true, then for some $\lambda_{0}>0$ and for some $n_{0} \in \mathbb{N}$ we must have $\lambda_{0}<a_{m} \leq a_{n}<2 \lambda_{0}$, therefore $\left(\bigcup_{i=1}^{\infty} K_{i}^{(m)}\right) \cap$ $\left(\bigcup_{i=1}^{\infty} K_{i}^{(n)}\right)=\emptyset$, for all $m>n>n_{0}$. If $i$ is large enough (that is $\left.\pi /\left(4 i^{2}\right)<\lambda_{0}\right)$, the ring $H_{i+1} \backslash H_{i}$ will not contain the $K_{j}^{(n)}$ for $n>n_{0}$ and for $j \in \mathbb{N}$. Thus, there is an index $i_{0}$ such that $K_{j}^{(n)} \subseteq H_{i_{0}}$ for all $n>n_{0}$ and for all $j \in \mathbb{N}$, which would imply $\lambda\left(H_{i_{0}}\right)=\infty$.

Let $\mathcal{H}^{\prime}$ be a maximal strongly laminar system in $\mathcal{S}$ containing $\mathcal{H}=\bigcup_{n=0}^{\infty} \mathcal{H}_{n}$. (The existence of $\mathcal{H}^{\prime}$ is guaranteed by Zorn's lemma.) It is easy to see that for any $S \in \mathcal{H}^{\prime} \backslash \mathcal{H}$, only case $(\gamma)$ of the proposition is possible. Furthermore, since $\kappa(S)=\lambda(S)$, there can be at most one such $S$ for any fixed $n$. Therefore, $\mathcal{H}^{\prime} \backslash \mathcal{H}$ is countable, and since $\mathcal{H}_{0}$ is countable, we obtain that $\mathcal{H}^{\prime}$ is countable. As it turns out, this construction leads to a system that is also maximal laminar (in addition to being maximal strongly laminar).

The above argument can be repeated for various other collections of sets including $\mathcal{S}=\{$ all closed rectangles (with sides parallel to the coordinate axes) $\}$.

\section{References}

[1] J. Baráth, P. Hajnal and E. K. Horváth, 'Elementary proof techniques for the maximum number of islands', European J. Combin. 32 (2011), 276-281.

[2] G. Czédli, 'The number of rectangular islands by means of distributive lattices', European J. Combin. 30 (2009), 208-215.

[3] G. Czédli, A. P. Huhn and E. T. Schmidt, 'Weakly independent subsets in lattices', Algebra Universalis 20 (1985), 194-196.

[4] S. Földes and N. M. Singhi, 'On instantaneous codes', J. Comb. Inf. Syst. Sci. 31 (2006), 317-326.

[5] E. K. Horváth, G. Horváth, Z. Németh and Cs. Szabó, 'The number of square islands on a rectangular sea', Acta Sci. Math. (Szeged) 76 (2010), 35-48.

[6] E. K. Horváth, Z. Németh and G. Pluhár, 'The number of triangular islands on a triangular grid', Period. Math. Hungar. 58 (2009), 25-34. 
[7] E. K. Horváth, B. Šešelja and A. Tepavčević, 'Cut approach to islands in rectangular fuzzy relations', Fuzzy Sets and Systems 161 (2010), 3114-3126.

[8] Zs. Lengvárszky, 'The minimum cardinality of maximal systems of rectangular islands', European J. Combin. 30 (2009), 216-219.

[9] Zs. Lengvárszky, 'The size of maximal systems of square islands', European J. Combin. 30 (2009), 889-892.

[10] Zs. Lengvárszky, 'Notes on triangular islands', Acta Sci. Math. (Szeged) 75 (2009), 369-376.

[11] Zs. Lengvárszky and P. P. Pach, 'A note on systems of rectangular islands: the continuous case', Acta Sci. Math. (Szeged) 77 (2011), 27-34.

[12] A. Máder and G. Makay, 'The maximum number of rectangular islands', The Teaching of Math. 14 (2011), 31-44.

[13] P. P. Pach, G. Pluhár, A. Pongrácz and Cs. Szabó, 'The possible number of islands on the sea', J. Math. Anal. Appl. 375 (2011), 8-13.

[14] G. Pluhár, 'The number of brick islands by means of distributive lattices', Acta Sci. Math. (Szeged) 75 (2009), 3-11.

[15] J. Thim, 'Continuous nowhere differentiable functions', Master's Thesis, Luleå University of Technology, 2003.

ZSOLT LENGVÁRSZKY, Department of Mathematics, Louisiana State University Shreveport, 1 University Place, Shreveport, LA 71115, USA

e-mail: zlengvar@1sus.edu 